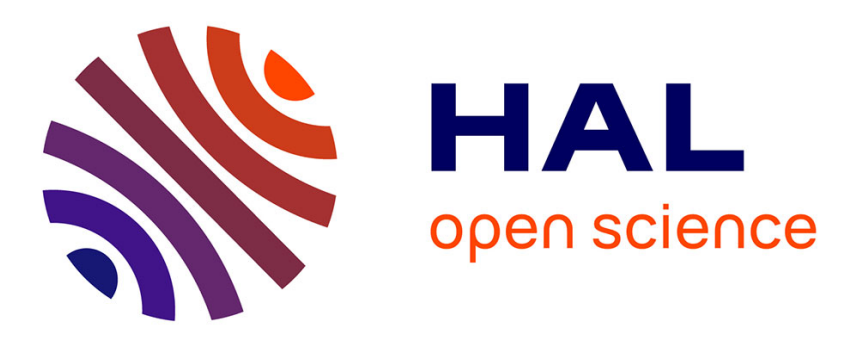

\title{
Assistive Systems for Mobility in Smart City: Humans and Goods
}

Yuhang Li, Chuantao Yin, Zhang Xiong, Bertrand T. David, René Chalon, Hao Sheng

\section{- To cite this version:}

Yuhang Li, Chuantao Yin, Zhang Xiong, Bertrand T. David, René Chalon, et al.. Assistive Systems for Mobility in Smart City: Humans and Goods. HCI in Mobility, Transport, and Automotive Systems, 12791, Springer International Publishing, pp.89-104, 2021, Lecture Notes in Computer Science, 10.1007/978-3-030-78358-7_6. hal-03363347

\section{HAL Id: hal-03363347 \\ https://hal.science/hal-03363347}

Submitted on 3 Oct 2021

HAL is a multi-disciplinary open access archive for the deposit and dissemination of scientific research documents, whether they are published or not. The documents may come from teaching and research institutions in France or abroad, or from public or private research centers.
L'archive ouverte pluridisciplinaire HAL, est destinée au dépôt et à la diffusion de documents scientifiques de niveau recherche, publiés ou non, émanant des établissements d'enseignement et de recherche français ou étrangers, des laboratoires publics ou privés. 


\title{
Assistive systems for mobility in Smart City: humans and goods
}

\author{
Yuhang $\mathrm{Li}^{1}$, Chuantao Yin ${ }^{1}$, Zhang Xiong ${ }^{2}$, Bertrand David ${ }^{3}$, René Chalon ${ }^{3}$ and Hao Sheng ${ }^{4,5}$ \\ ${ }^{1}$ Sino-French Engineer School, Beihang University, 100191, Beijing, China \\ yuhang.li@buaa.edu.cn, chuantao.yin@buaa.edu.cn \\ ${ }^{2}$ School of Computer Science and Engineering, Beihang University, 100191, Beijing, China \\ xiongz@buaa.edu.cn \\ ${ }^{3}$ Université de Lyon, CNRS, Ecole Centrale de Lyon, \\ LIRIS, UMR5205, F-69134, Lyon, France \\ Bertrand.David@ec-lyon.fr, Rene.Chalon@ec-lyon.fr \\ ${ }^{4}$ State Key Laboratory of Software Development Environment, School of Computer Science and Engineering, \\ Beihang University, 100191, Beijing, China \\ ${ }^{5}$ Beijing Advanced Innovation Center for Big Data and Brain Computing, Beihang University, 100191, Beijing, \\ China \\ shenghao@buaa.edu.cn
}

\begin{abstract}
Nowadays, the society is highly intelligent, and smart city has become the common expectation of people. Smart city is a large concept, which includes smart home, Smart Transportation, Smart public, Service and Social management, smart Urban Management and other aspects. This paper mainly discusses the problem of the mobility in cities. The mobility in cities can be divided into two categories in practice, namely, the movement of humans and the movement of goods. In this paper, we enumerate the typical cases in the mobility of humans and goods, and analyze the operation principle of these cases one by one. Some of these examples are implemented, some are not. In order to make urban mobility "smart", we propose a framework of assistive system, which has a four-tier structure and uses big data, cloud computing, AI, deep learning and other technologies. It is aimed at a variety of different scenes, to provide users with the most thoughtful advice and the most humanized service, to solve the problem of urban mobility
\end{abstract}

Keywords: Smart City, mobility of humans, mobility of goods, Assistive systems

\section{Introduction}

\subsection{Background}

The rapid development of information technology has brought about a global trend of informatization. In the future, it is increasingly necessary to rely on information technology to promote the development of smart cities. Governments around the world have spontaneously proposed a plan to rely on the Internet and information technology to change the city's blueprint 
for the future.

The United States took the lead in proposing the National Information Infrastructure (NII) and global Information Infrastructure (GII) programs. Then, the European Union focused on promoting the Information Society program and identified ten application fields of the European Information society as the main direction of the construction of the Information society of the European Union [1-2].

As the whirlwind of smart cities intensifies, countries are constantly putting innovative ideas into action. In the process of industrial transformation and social development, many developed countries, such as the United States, the United Kingdom, Japan, the Netherlands, Sweden, South Korea, Singapore and Malaysia have recognized the importance of smart cities earlier and have achieved remarkable results [3-4]. At present, countries all over the world have different degrees of realizing the idea of smart cities and different implementation efforts. However, there is no denying that in the 21 st century, the age of smart has come.

\subsection{What is smart city?}

The term 'smart city' appeared for the first time in the early 1990s, and researchers have emphasized technology, innovation and globalization in the process of urbanization [5]. Smart cities have attracted great attention since 2008, with the launch of IBM's Smarter Planet project [6]. Since then, the concept of smart cities has continued to grow and evolve.

Smart city originated in the field of media, which refers to the integration of urban systems and services by using various information technologies or innovative concepts, so as to improve the efficiency of resource utilization, optimize urban management, and improve the quality of life of citizens.

As a new mode and concept of modern city operation and governance, smart city is established on the basis of complete network infrastructure, massive data resources, multi-domain business process integration and other information and digital construction, which is an inevitable stage in the development process of modern city.

Smart city often intersects with regional development concepts such as digital city, perceptive city, wireless city, ecological city and low-carbon city, and even gets mixed up with industrial digitalization concepts such as e-government, intelligent transportation and smart power grid. People's interpretation of the concept of smart city often has different emphasis, some thought the key lies in the application of technology, some thought that the key lies in the network construction, some view is the key in the participation of people, some thought that the wisdom is the most important [7-11]. In a word, smart is more than intelligence. Smart city is not only another term for intelligent city, but also includes people's intelligent participation, people-oriented, sustainable development and other connotations.

\subsection{Mobility in smart city}


Smart City is a very large concept, which contains many sub-fields. Su et al. [12] summarized eight main applications of Smart City in real life (shown in Fig. 1): wireless city, smart home, smart transportation, smart public service and social management, smart urban management, smart medical treatment, green city and smart tourism. Figure 1 shows the construction frame of application systems for smart city.

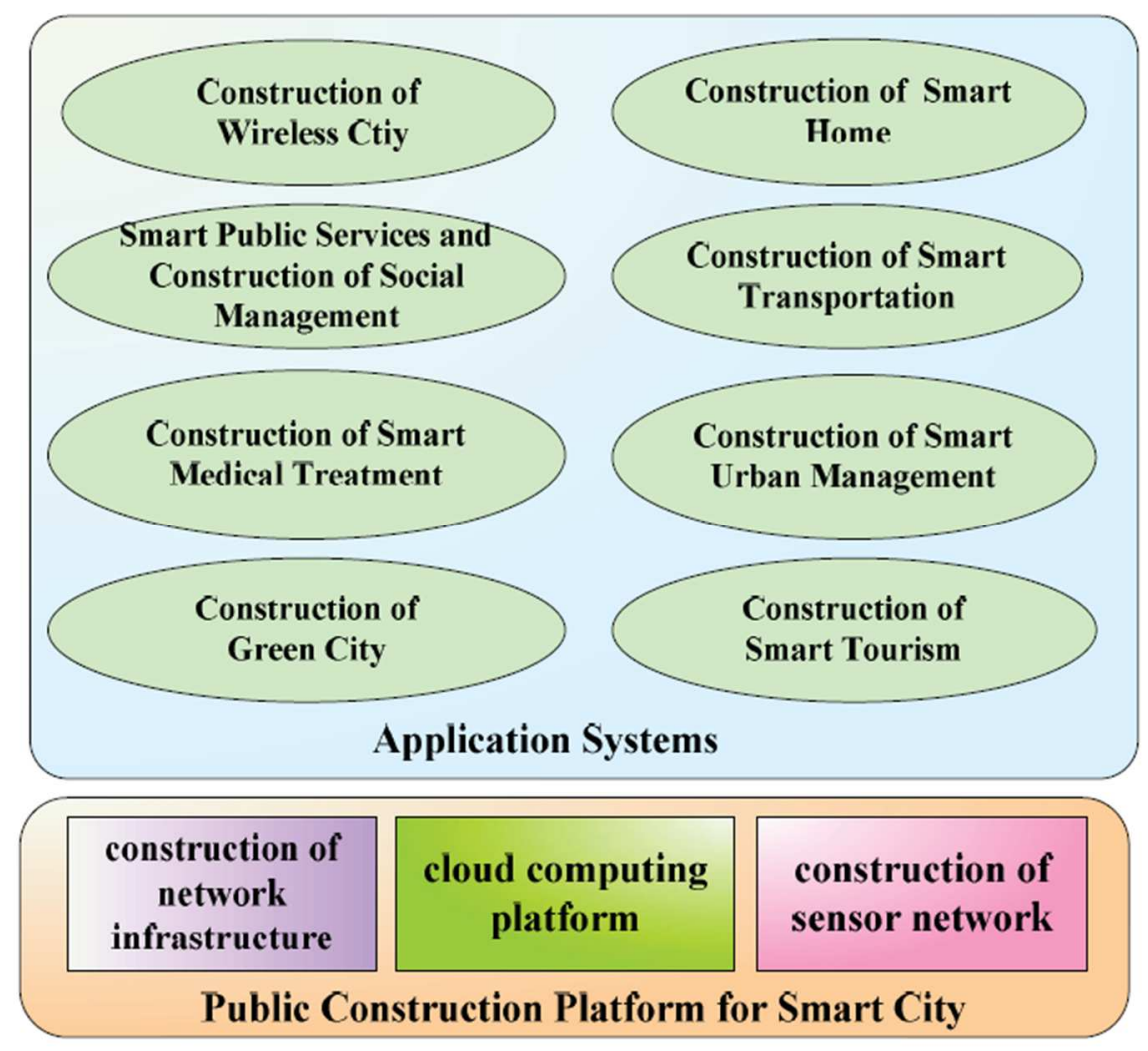

Figure 1: Construction Frame of Application Systems for Smart City

The architecture of smart cities is too large and it contains too many fields, so we cannot explain them all in detail in one article. Therefore, in this article, we mainly explore a sub-problem of the smart city, that is, the problem of urban mobility, which includes goods or humans with or without special needs. The description of other applications is available in [13].

\section{Assistive system of smart city}

\subsection{Intermediation platform: a state-of-the-art assistive system}

The mobility in cities is mainly devoted to two aspects: the mobility of people and the mobility of goods. Every second, thousands of people or goods in cities need to move. For people, there are many problems arising from the demand of mobility, such as the travel itinerary, route planning, how to park, and so on. Even things get more complicated when the people who travel are disabled. For the goods, how to choose the carrier is an obvious problem, in addition to this, the transfer of goods between different carriers and deliverymen cannot be ignored neither. It is very important to arrange and dispatch the traffic resources and human resources of city reasonably[14]. 
Based on these problems, we seem to need an intermediation platform to intelligently collect the data generated during the mobility. Through the analysis and simulation of these data, we can use scientific and information-based ways to meet our mobility needs. This platform can help us to make the fastest and most reasonable choice.

\subsection{Architecture of the intermediation platform}

In order to meet our requirements, we need to construct a four-layer intermediation platform, including sensing layer, communication layer, data layer and application layer (Fig.2).

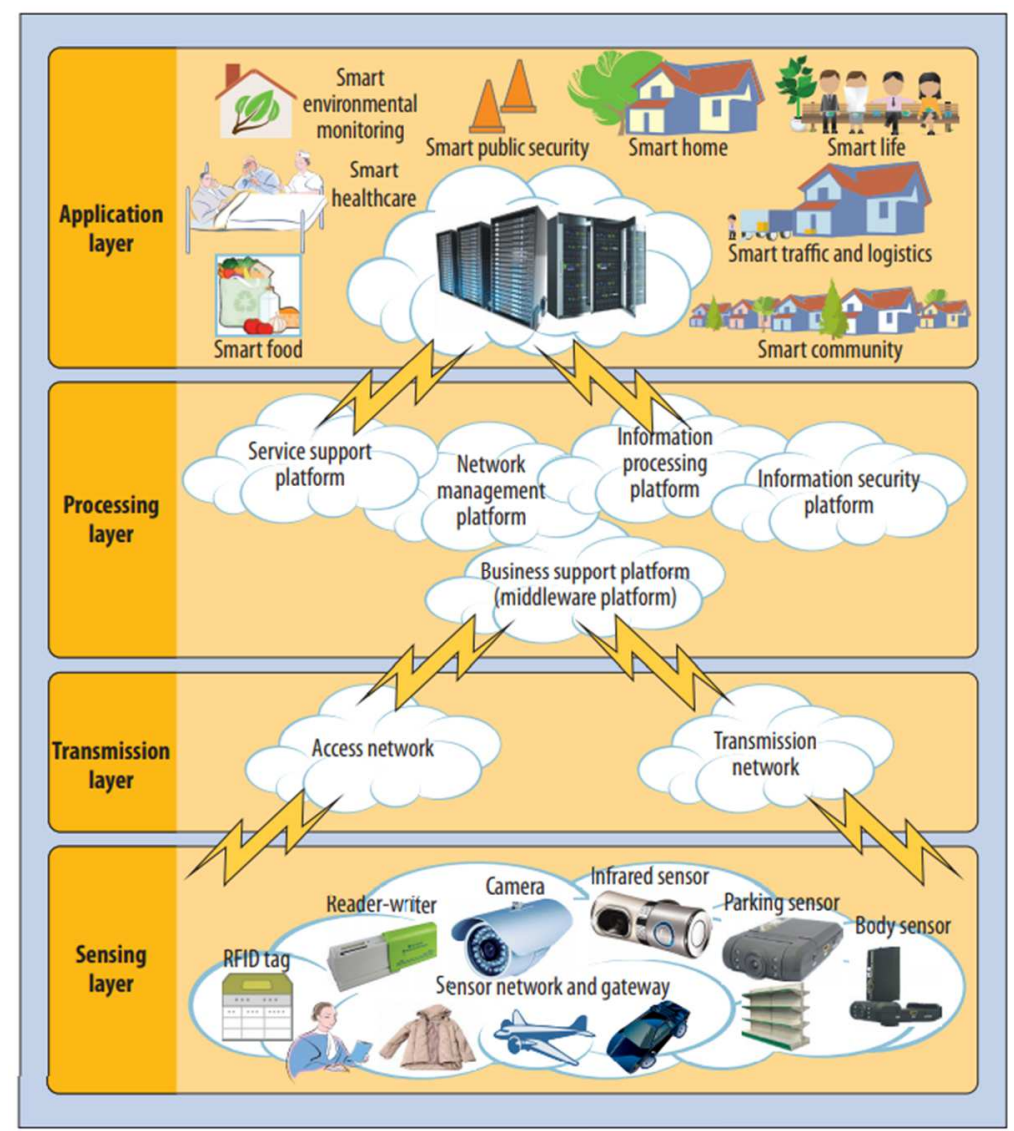

Figure 2: Smart city architecture summarized by Liu and Peng [24]

\section{Sensing layer}

The sensor layer has IOT(Internet of Things) nodes all over the city, which is composed of sensors, microchips, power supplies and networks. It is responsible for collecting the data of various activities in the physical environment, and then sending the data to the data center through the data layer.

\section{Communication layer}

Each intelligent city system has at least 1 billion IOT nodes distributed throughout the city. Because a large number of IOT nodes are distributed in a city, it is necessary to establish a reliable communication technology, which covers a wide geographical area and can handle a large amount of data flow, it becomes necessary to transmit hundreds of millions of data flows to the central server quickly, safely and stably.

\section{Data layer}

The foundation of data layer is a data server which processes data with different data models. 
These data models includes prediction model, description model, decision model and so on. These models use big data, cloud computing, AI, deep learning and other means to analyze data and give reasonable suggestions. With the help of these models, the municipal government can make forward-looking decisions based on the data.

\section{Application layer}

As a visual platform that can directly interact with users, the application layer is the only level that is presented to the public. Typically, on the phone, the application layer is shown as apps. Users can enter their own mobility requirements, such as planning a destination, to get the desired path recommendation.

In general, when we talk about an intermediation platform, we only mean the application layer in a four-tier structure. The other three layers are invisible, but they support the application layer. To put it simply, for every citizen, our assistive system is a collection of a series of apps, that is, a multi-functional intermediation platform, which provides users with the most thoughtful suggestions and the most humanized services for various scenarios, so that people and goods can flow without barriers.

Without massive data and complex algorithms, our intermediation platform would be a beautiful shell. These invisible structures require huge investment in infrastructure and human resources, which each country needs to realize step by step according to the actual situation.

\section{Mobility of humans and goods}

In the remainder of this article, we will focus on some of the classic examples of urban mobility in our lives, some of which have already been achieved, and some of which are not yet. We analyze the essential problems in each case and propose appropriate solutions through our mediation platform.

\subsection{Mobility of humans}

\subsubsection{Intelligent navigation}

Intelligent navigation, as a scenario achieved, the main solution is the path planning problem. When the user opens the app, the app will first locate the current location through GPS satellites. After entering the destination, the platform will plan an optimal route according to the user's starting position, destination location and travel mode.

Since road conditions in reality need to consider more factors such as road condition and environment, the "optimal" path obtained by the basic algorithm based on the graph is not necessarily the one that users want most. At this time, the navigation provider may give some suboptimal paths to add to the search results and provide users with choices. And then learn from the feedback of users, so as to get the path planning results more in line with users' needs.

One example is that the navigation system gives the user several different routes to choose from, including the shortest route, the least transfer, and so on. In addition, after the retrieval results are obtained in the navigation system, users can "drag" the route on the map to get a more appropriate route. The information of these users "drag" will be sent to the server for saving, so as to make recommendations to other users later [15-17]. 
In the case of real-time navigation, things get more complicated. For example, when a user drives a private car to travel, the navigation system needs to monitor real-time traffic flow, real-time weather conditions, accident sections and other information, and optimize the path at any time by combining this information, and provide information to the driver through variable information board or voice prompt. This requires a data acquisition system to monitor and control traffic conditions. The collection of these data mainly relies on vehicle detector system, weather station, visibility detector and other equipment to complete.

In this case, the sensor layer is the data acquisition system that we just mentioned. It can collect data and monitor in real time to provide information for the whole system. Communication layer is a communication network, which is used to transmit a large number of data. The data layer integrates all data, combines with real-time traffic information, and proposes the best solution. Finally, the application layer, as a tool to communicate with users, transmits the recommended results to users by voice broadcast.

\subsubsection{Smart parking}

The smart parking system can effectively solve the city parking difficult problem. In urban parking, the owner only needs to use a smart parking app to realize all the functions needed for parking. The owner can check the vacant parking space in the designated parking lot at any time and make an online reservation. In addition, when the owner enters a destination location, the smart parking system will show him or her all available parking places near the destination and the number of available parking spaces in each location. For car owners who are not familiar with the parking routes, the APP also provides parking lot navigation services to help everyone find available parking spaces. The system also supports the online payment service, the car owner can leave the parking lot directly after the end of the parking, and pay online. This saves time in checkout lines and greatly improves the efficiency of parking lots. The following is the overall structure of the intelligent parking system:

\section{Intelligent hardware layer}

The terminal intelligent device is the infrastructure for the operation of the city-level parking management system, providing parking services for users and business data for the platform.

\section{Network layer}

The network is the communication foundation of the whole Internet. For the on road parking service, one of the core business of urban parking, using loT network has the characteristics of large connection, low power consumption, low cost and wide coverage, and its features of simple installation and high reliability are also more conducive to the construction and maintenance of on road parking projects.

\section{Platform layer}

Platform layer is an open integration platform with multiple API interfaces, which is responsible for access management of network layer information and integration analysis of data.

\section{Operation layer}

The operation layer is the core business layer of the urban parking platform, including subsystems such as in-road, off-road, induction, monitoring, member account, payment and settlement, marketing incentives, statistical analysis, operation management, information release, etc. The operation layer must fully realized the integrated business capability of parking business, constructed diversified payment channels, integrated member, payment, settlement, marketing and 
other links, and realized the closed-loop function of online and offline scenes.

\section{Service layer}

Smart parking app, public account, background management system and open interface of resources provide more intelligent, more convenient and more efficient parking management services for car owners, organizations and businesses.

\subsubsection{Smart taxi-hailing "Didi Chuxing"}

An example of smart taxi-hailing is the Didi Chuxing App, which has changed the traditional way of taxi-hailing and established and cultivated a modern way of travelling led by users in the era of big mobile Internet. Compared with the traditional telephone call and roadside call, the birth of didi taxi has changed the traditional taxi market pattern, overturned the concept of roadside stop. Using the characteristics of mobile Internet, it integrates online and offline, from the initial stage of taxi to the use of online payment. It maximizes the passengers' taxi experience, changes the traditional taxi driver and other ways, and makes the driver more comfortable According to the passenger's destination, the master "receives the order" according to his will, which saves the communication cost between the driver and the passenger, reduces the empty driving rate, and saves the resources and time of the driver and the passenger as much as possible.

Users can use the Didi Chuxing app to book trips and use online payment at the end of the trip. In the process of taking a taxi, the user enters the starting point and destination, and the app uploads the user's request to the driver-pickup platform and recommends it to eligible drivers nearby. Those who are willing can grab the order online. After receiving the order successfully, the user can see the driver's car model, license plate number and driver's phone number in the app. The two parties can contact each other through the phone to further communicate about service requirements. At the same time, the user can also know the driver's real-time geographical location, as well as the estimated time of arrival to the starting point.

Didi Chuxing's drivers are not limited to full-time drivers. As long as the owners meet the requirements, they can register as a driver on the platform. The conditions are as follows:

Age: 21-60 for males and 21-55 for females, with small variations depending on the city.

Driver's license: Drivers should have relevant driving license and at least 3 years of driving experience.

Drivers need: no criminal record, no traffic accident crime, no criminal record of dangerous driving, no drug record, no record of drunk driving.

On the one hand, Didi Chuxing enables car owners to make money by taking orders in their spare time; on the other hand, it enables users to reach their destinations quickly and efficiently at a lower price, which is convenient for both parties. This is a typical case of urban mobility in the smart city concept.

\subsubsection{Bike-sharing}

Another typical case is shared bikes. Bike-sharing refers to the way in which enterprises provide bike-sharing services in campuses, subway stations, bus stations, residential areas, business districts and public service areas. It is a time-sharing rental model. This is also a new kind of environmental sharing economy.

At present, the existing transportation system cannot fully meet people's travel needs. Even in 
first-tier cities with well-developed transportation networks, there is still a shortage of short-haul transportation. Taking public transportation or driving private cars is not the most convenient way for people to travel between 1 and 3 kilometers. Because of its small size, easy to park, convenient and quick to use, environmentally friendly characteristics, the bicycle can perfectly adapt to the travel needs. In response to this phenomenon, shared bikes have emerged.

The operation method of renting shared bikes is very simple: users need to pay a deposit of 99 299 yuan and get the right to use the bikes when they log into the APP client through their mobile phone number. Then, the vehicle location information is used to find the parked vehicle nearby, scan the code or enter the body number manually to open the lock, and then start riding. The app will start the timing after the lock is opened. After cycling, users need to park their bikes in a safe position on the roadside and re-lock them. When the user clicks "End of ride" in the app, or when the app recognizes that the vehicle is relocked, the ride ends and the billing ends. Fees are calculated according to the ride time, 0.1 to 1 yuan per 30 minutes, if less than 30 minutes will be charged according to the basic price.

Bike-sharing offers users an excellent experience due to its convenience, environmental friendliness, economic benefits and high degree of freedom. However, shared bikes are currently facing too little profit. At present, the main source of revenue for bike-sharing companies is the rental of a single bike. If the cost of a bike is 3,000 yuan, renting it four to five times a day with each rental being 1 to 2 yuan, it will take about two years for a bike to recover its cost. The bicycle itself has a short life, which is easy to cause damage. The fluctuation of car demand caused by bad weather and other factors is also inevitable. Therefore, the current profit model of shared bikes cannot generate enough revenue, and enterprises must find a more suitable profit model to continue the development of this sharing economy model.

\subsubsection{Travel for the disabled}

For the disabled, travel is much more difficult than that of normal people. Normal people only need to consider travel mode, travel route, destination and other information, while disabled people need to consider more information. For example, for blind people, our auxiliary system is their "eyes". The user can activate the navigation by voice. The system receives the voice message, plans the route and announces the voice. The GPS module can locate the starting position and destination position of the blind person, but such positioning is biased and not accurate enough to carry out accurate positioning. Therefore, the auxiliary system should be equipped with an ultrasonic module, which can measure obstacles on the path of the blind through ultrasonic waves and inform the blind of the specific location of obstacles by voice, so that the blind can effectively avoid obstacles.

However, for people with physical disabilities and mobility difficulties, the route planning during travel needs to take into additional consideration whether the site has barrier-free access. For people with limited mobility, going up and down stairs is extremely inconvenient, especially in the case of solo travel, so the need to use the elevator floor movement. Therefore, our system needs to establish a sound message network to obtain timely information about the availability of barrier-free channels, and then give a plan.

Considering that the users may be disabled, our assistive system needs to add a depth assistance mode. When logging into the app for the first time, the user can make a choice and switch the navigation mode at any time. When using depth assistance mode, the system will make more 
detailed path recommendation for specific types of users, including barrier free navigation, voice navigation and so on.

\subsubsection{Travel planning}

In order to enrich the demand of urban mobility, we can also consider the problem of travel planning. It belongs to the part of smart tourism in the structure of smart city, but also has an inseparable connection with urban mobility. By developing a smart travel app, we can provide users with personalized and personalized travel recommendations. When users log in the app for the first time, they can select travel tags they are interested in, such as "Beach" and "Aurora", etc. According to these tags, the platform can recommend destinations and routes to users through the recommendation algorithm. The travel plan obtained by the user includes the location of the play, the recommended time of play for each place, the recommended play items, the transportation to get from one scenic spot to the next, and so on. Users can also upload their own travel routes to the app at the end of a trip, as an alternative route recommended by the app.

Through cooperation with travel agencies, the App can include all kinds of popular travel routes as alternatives. If users determine travel routes through the App, they can enjoy preferential prices in cooperation with the platform. Similarly, the hotel users choose to cooperate on the platform can also enjoy platform discounts. Through this app, users will have a more affordable and convenient travel experience.

In order to make recommendations, the platform needs to use the recommendation algorithm in the field of deep learning. By analyzing the tags the user chooses to be interested in and his or her browsing history, the recommendation algorithm can calculate the similarity between users or between travel items to make recommendations. After a user has planned many trips through the app, the platform can extract the user's travel preferences to build the profile portrait of the user, and then make more accurate recommendations.

\subsection{Mobility of goods}

Another aspect of urban mobility is the movement of goods. Unlike human movement, the movement of goods is usually passive and based on human will. In reality, the movement of goods needs to be supervised by a special person, that is, the movement needs to be carried out under supervision. Then the route of cargo transport, the way of movement and the planning of the custodians need to be carefully considered. Next, we will analyze several typical cases of the flow of goods, for example, takeaway delivery problems, supermarket order and delivery problems, long-distance mail problems and so on.

\subsubsection{Food delivery}

With the impact of the epidemic worldwide, people are more and more inclined to enjoy food without leaving their homes. As a result, the food delivery industry has made great progress. The process of producing and finishing a complete takeout order is as follows.

First, the user browses the takeaway app, selects the food he or she wants, submits the order, confirms the address, pays online, and finally places the order successfully. After placing the order successfully, the merchant will receive the order notice. The merchant can choose whether to 
accept the order or not. If not, fill in the reason for rejection and inform the user that the order has been canceled. If the order is received, the user is notified of the receipt. When the merchant makes food, the system selects the rider who is free within a certain range and sends an order to him or her through the algorithm. If the rider refuses, the next rider in line will be notified. When there is a shortage of riders at the peak of meal, the system will plan the route for each rider to pick up and deliver food, find the optimal solution and inform the corresponding rider to pick up food. When multiple order recipients are close to each other or destination, the system will give priority to recommending the same rider to receive orders to save manpower. After receiving the order, the rider picks up the goods at the store and starts distribution. After the order is completed, the system notifies the user for delivery, and finally the user makes evaluation. During the delivery process, each step will be reported to the ordering user through the delivery app in real time. In the final docking stage between the rider and the ordering user, the rider will contact the user by phone and determine the specific place to pick up the meal.

In this case, we used a synchronous solution to the distribution problem. Due to the limited nature of the food itself, the rider and the ordering user usually meet face to face.

\subsubsection{Supermarket delivery}

Different from the previous case, when the distribution of goods from food to daily necessities, the distribution mode can also be changed accordingly. In addition to the synchronous solution mentioned in the previous case, which is perfect for this case, we can also use the asynchronous approach to solve the distribution problem. The asynchronous means that in the process of distribution, the delivery personnel and the ordering personnel cannot appear at the same place, face to face, but choose to temporarily ship the goods to a designated location, the delivery personnel will deliver the goods to the designated location and notify the orderer, who will pick up the goods at their own time.

We are currently working on an auxiliary system based on the sharing economy to address pick-up needs by setting up a special supermarket aisle. The supermarket publishes the goods online for consumers to choose and buy. The consumers submit the order and fill in the expected pick-up time, and then go to the supermarket to pick up the ordered goods in their spare time. Supermarket aisles are usually located near the supermarket and organized to minimize loading time.

The supermarket aisles, which is open 24 hours a day, includes a supermarket lane and a supermarket sidewalk, which are designed for different needs. Among them, the supermarket lane is designed for consumers driving private cars. Consumers can get the ordered goods without getting off the bus, which greatly reduces the time cost. The supermarket sidewalk is designed for walking consumers, which helps users save a lot of shopping and queuing time [18].

Fresh products require special consideration because they will not last long without being refrigerated. If the consumer can pick up the goods quickly, then only need the merchant to carry out simple short-term preservation treatment, such as vacuum storage, add ice bags, etc. But if the consumer is unable to get the goods in a short time due to objective reasons, then they need to use the refrigerator. Refrigerated cabinets, provided by supermarkets, are specially designed to store fresh products and ensure that consumers can get the freshest goods at any time. 


\subsubsection{Parcel post}

Both of these cases are examples of short-haul freight. Whether it's takeout or supermarket orders, the goods are moving only within a city, or less. In this case, the delivery time of the goods and the pickup time of the orderer are predictable. But if our goods move to different cities, or even to different countries, the arrival time of goods becomes difficult to estimate in the case of long distance. This situation makes synchronous solutions no longer applicable, and we need to use asynchronous solutions to address these types of requirements.

When a user places an order online and buys an item from a store on the Internet, the merchant confirms the address to the consumer again, and arranges the delivery after confirming it is correct. Each cargo is marked with a unique QR code, which will be scanned and entered into the system when passing through each large distribution point and made available to consumers for inspection.

When the package is about to arrive at the final distribution center, the intermediary platform needs to obtain all the collection points registered in the system near the delivery address and further filter them to finally select the appropriate transporter.

The process of discovering a transporter who is a neighbor of the ordering client (receiver) can be based on different situations. The first is a totally open situation in which the goal is to find and create dynamically an association by checking the proximity of geographical locations between ordering persons and potential transporting persons. The presence of potential transporting persons can be discovered either by explicit declaration (signaling of presence in the shop or shopping mall) or by contextual location detection (by smartphone localization or other implicit identification). In this case, the intermediation algorithm must be able to take into account a huge amount of data.

In a more collaborative situation, parcel transportation can be an interesting functionality of a neighbor association collaborative system or an intergeneration collaborative system in which interpersonal help, information and cooperation are supported. Through this system, its members have access to a list of members and can either pre-establish potential relationships between the ordering person and the transporting person (one or more) or have access to less accurate information providing a list of potential transporting persons. These data can be supplied to the delivery service of the shop or mall. In this case, the intermediation algorithm works on a limited set of data [19].

When the package arrives, the system will send a message to the consumer, reminding him or her to pick it up as soon as possible. 


\section{Deep learning approach in assistive system}

In some situations, it seems that deep learning can be used to analyze existing data and lead to interesting solutions. We can take intelligent navigation and travel planning as examples. For intelligent navigation, the intermediation platform considers the starting position and the destination position, combines the time information, meteorological information and other interfering factors, and finally provide the most appropriate one or several recommended paths. As for travel planning, the focus is to intelligently analyze the user's intention of his or her behavior and finally make travel recommendations based on the tags he is interested in and his browsing history.

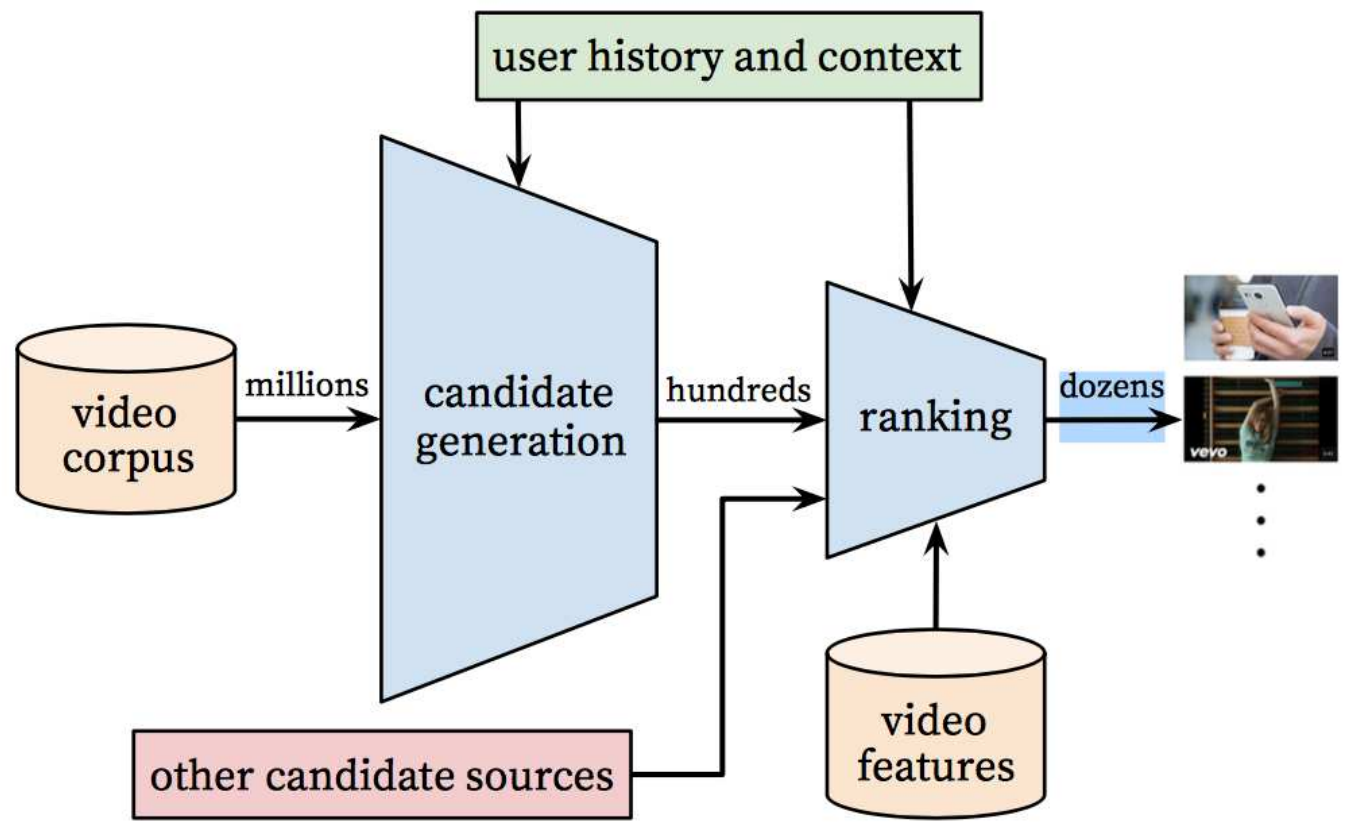

Figure 3: Structure of recommendation system algorithm

Deep learning is hugely popular today. The past few decades have witnessed its tremendous success in many applications. Academia and industry alike have competed to apply deep learning to a wider range of applications due to its capability to solve many complex tasks while providing state-of-the-art results [20]. Recently, deep learning has also revolutionized intermediation architectures, providing more opportunities to improve matching performance. Recent advances in deep learning-based intermediation platforms have gained significant attention by overcoming obstacles of conventional models and achieving high recommendation quality. Deep learning catches the intricate relationships within actual data, from abundant accessible data sources such as contextual, textual and visual information.

The classical recommendation system algorithm consists of two parts: one for candidate generation and one for sorting. As shown in the figure 3, taking video recommendation as an example and users' browsing history as input, candidate generation network can significantly reduce the number of recommended videos and select a group of most relevant videos from a large library. This generates candidate videos that are most relevant to the user, and then we predict the 
user ratings and recommend one or more of the highest scores to the user.

First, some shallow distributed representation models are introduced. At present, the shallow distributed representation model has been widely used in the field of text, such as Word2VEc, GloVec, etc.. In contrast to the traditional model, the word embedding model can map words or other information units (such as phrases, sentences, documents, etc.) into a low-dimensional implicit space. In this implicit space, the representation of each information unit is a dense eigenvector. The basic idea of the embedded word representation model is actually from the traditional "Distributional semantics", which can be summarized as the semantic meaning of the current word is closely related to its adjacent background words. Therefore, the modeling method of word embedding is to use embedded representation to construct the semantic association between the current word and the background word. Compared with the multi-layer neural network, the training process of word embedding model is very efficient, with good practical effect and good interpretibility, so it has been widely used.

Corresponding to the neural network model, the most common models include multilayer perceptron[21], convolutional neural network[22], cyclic neural network[23], recursive neural network[24], etc. The multi-layer perceptron mainly uses the multi-layer neuron structure to construct the complex nonlinear feature transformation. The input can be various extracted features, and the output can be the label or value of the target task. In essence, a complex nonlinear transformation can be constructed. A convolutional neural network can be directly deployed on a multi-layer perceptron. The input features of the perceptron are likely to be indefinite or ordered. Through multiple convolutional layers and sub-sampling layers, a vector of fixed length is finally obtained. Cyclic neural network (NN) is a common model used for modeling sequential sequences, describing the correlation of implied states and capturing the data characteristics of the whole sequence. To solve the problem of long-term dependence (" gradient disappearance ") on simple cyclic neural networks, which cannot make use of long interval historical information effectively, two improved models are the long and short time memory neural network (LSTM)[25] and the cyclic unit (GRU) based on gate mechanism. Recursive neural network can be considered as a simplified recursive neural network, which recurses continuously to obtain a sequence representation according to a given external topology.

In practice, we can use RNN structure in path planning, including intelligent navigation, travel for the disabled, Parcel post and so on. And CNN model can be used for vehicle recognition to assist smart parking. For some scenarios with recommendation tasks, such as goods transportation and travel planning, we can use multi-layer perceptron, LSTM and other models.

Deep learning-based intermediation has proved very effective. However, the key problem for deep learning in different application scenarios is always data. Once data have been sufficiently collected and tagged, some deep learning-based solutions could be studied and applied.

\section{Conclusion}

In this paper, we mainly study the mobility in smart cities. First of all, we discuss the birth and development of smart cities in today's society. Smart city is a very big concept, which includes a series of fields such as Smart home, Smart Transportation, Smart public, Service and Social Management, smart Urban Management, etc. We cannot discuss each aspect in detail. Therefore, in this paper, we choose the issue of urban mobility for further in-depth discussion, which is also 
the focus of this paper.

Subsequently, in order to make the mobility of the city "smart", we proposed a framework of assistive system with a four-layer structure, which was concretely transformed into apps with different functions in the final application layer. To put it simply, for every citizen, our assistive system is a collection of a series of apps, that is, a multi-functional intermediation platform, which provides users with the most thoughtful suggestions and the most humanized services for various scenarios, so that people can travel barrier-free.

The mobility in cities can be divided into two categories in practice, namely, the mobility of humans and the mobility of goods. In this paper, we enumerate the typical cases in the flow of humans and goods, and analyze the operation principle of these cases one by one. Among them, there are 6 situations of human mobility, and the flow of goods is divided into 3 situations. Some of these examples have already been implemented, and some are the way forward.

Finally, according to the different scenarios that have been analyzed, the algorithm models that can be used for the assistive system are presented, from the shallow distributed representation model to the more complex neural network model.

From the case study, we can see that one of the most basic requirements is the popularity of online payment. Only when online payment is really popularized, can people's life gradually develop towards informatization and digitization. Secondly, in order to achieve various algorithms, data is really indispensable, and in order to obtain data, it needs a series of supporting infrastructure. Under the blueprint of sharing economy, everyone is not only the beneficiary of smart city, but also the provider of data.

Acknowledgements. This study is partially supported for Chinese part by the National Key R\&D Program of China (No. 2019YFB2102200), the National Natural Science Foundation of China (No. 61977003, No. 61861166002, No. 61872025, No. 61635002), the Science and Technology Development Fund of Macau SAR (File no. 0001/2018/AFJ) Joint Scientific Research Project, the Macao Science and Technology Development Fund (No. 138/2016/A3), the Fundamental Research Funds for the Central Universities and the Open Fund of the State Key Laboratory of Software Development Environment (No. SKLSDE2019ZX-04). For French part main supports were by the French Ministry of Ecology (No. 09MTCV37 and PREDIT-G02 CHORUS 2100527197).

\section{References}

[1] Bronstein Z. Industry and the smart city. Dissent, 2009, 56: 27-34

[2] Digital Agenda Scoreboard 2015: Most targets reached, time has come to lift digital borders. Website of Digital Agenda for Europe. http://ec.europa.eu/digital-agenda/en

[3] Hosaka T A. Japan creating 'smart city' of the future. San Francisco Chronicle. Associated Press, October 11, 2010

[4] Ng P T. Embracing emerging technologies: the case of the Singapore Intelligent Nation 2015 Vision. In: de Pablos P O, Lee W B, Zhao J Y, eds. Regional Innovation Systems and Sustainable Development: Emerging Technologies. Hershey: IGI Global, 2011. 115-123

[5] Gibson D V, Kozmetsky G, Smilor R W. The Technopolis Phenomenon: Smart Cities, Fast 
Systems, Global Networks.Rowman \& Littlefield Publishers, 1992

[6] Palmisano S J. A smarter planet: the next leadership agenda. IBM, 2008

[7] Ginger R, Gudrun H. Smart cities ranking: an effective instrument for the positioning of the cities? Architecture. City Environ, 2010, 4: 7-26

[8] Washburn D, Sindhu U, Balaouras S, et al. Helping CIOs understand 'smart city' initiatives. Growth, 2009, 17

[9] Su K, Li J, Fu H. Smart city and the applications. In: Proceedings of IEEE International Conference on Electronics, Communications and Control (ICECC), Ningbo, 2011. 1028-1031

[10] Mitton N, Papavassiliou S, Puliafito A, et al. Combining Cloud and sensors in a smart city environment. EURASIP J Wirel Commun Netw, 2012, 2012: 1-10

[11] Nam T, Pardo T A. Conceptualizing smart city with dimensions of technology, people, and institutions. In: Proceedings of 12th Annual International Digital Government Research Conference: Digital Government Innovation in Challenging Times. New York: ACM, 2011. 282291

[12] Su K, Li J, Fu H. Smart city and the applications. In: Proceedings of IEEE International Conference on Electronics, Communications and Control (ICECC), Ningbo, 2011. 1028-1031

[13] Yin C., Xiong Z., Chen H., Wang J., Cooper D., David B.: A literature survey on smart cities. Science China, Information Sciences, October 2015, Vol. 58 100102:1-100102:18, Science China Press and Springer-Verlag Berlin Heidelberg 2015 info.scichina.com link.springer.com (2015). doi: 10.1007/s11432-015-5397-4

[14] Gonzalez H, Han J, Li X, et al. Adaptive fastest path computation on a road network: a traffic mining approach. In: Proceedings of 33rd International Conference on Very Large Data Bases, Vienna, 2007. 794-805 Chuantao Yin, Bertrand David, René Chalon, Hao Sheng: Assistive Systems for Special Needs in Mobility in the Smart City. In: HCI in Mobility, Transport, and Automotive Systems. Driving Behavior, Urban and Smart Mobility - Second International Conference, MobiTAS 2020, Held as Part of the 22nd HCI International Conference, HCII 2020, Copenhagen, Denmark, July 19-24, 2020, Proceedings, Part II. Lecture Notes in Computer Science 12213, Springer, 376-396

[15] Ziebart B D, Maas A L, Dey A K, et al. Navigate like a cabbie: probabilistic reasoning from observed context-aware behavior. In: Proceedings of 10th International Conference on Ubiquitous Computing. New York: ACM, 2008. 322-331

[16] Li B, Zhang D, Sun L, et al. Hunting or waiting? Discovering passenger-finding strategies from a large-scale real-world taxi dataset. In: Proceedings of IEEE International Conference on Pervasive Computing and Communications Workshops, Seattle, 2011. 63-68

[17] Sun L, Zhang D, Chen C, et al. Real time anomalous trajectory detection and analysis. Mob Netw Appl, 2013, 18:341-356

[18] David B., Chalon R.: Box/Lockers' contribution to Collaborative Economy in the Smart City. 2018 IEEE 22nd International Conference on Computer Supported Cooperative Work in Design (CSCWD 2018), 11 May 2018, Nanjing (Chine), pp. 802-807 (2018). doi: 10.1109/CSCWD.2018.8465151.

[19] David B., Chalon R., Yin C.: Collaborative systems \& Shared Economy (Uberization): Principles \& Case Study. EEE'16 - The 2016 International Conference on e-Learning, e-Business, Enterprise Information Systems, and e-Government, 28 July 2016, Las Vegas, Nevada (US), pp. 134-140. (2016) HAL: hal-01496630. 
[20] Paul C, Jay A, Emre S.: Deep neural networks for YouTube recommendations. In Recsys. 191-198 (2016)

[21] Dziugaite G. K., Roy D. M.: Neural network matrix factorization, arXiv preprint arXiv: 1511.06443 (2015).

[22] Tomas Mikolov. Using Neural Networks for Modeling and Representing Natural Languages. COLING (Tutorials) 2014: 3-4

[23] TANG Jiaxi, WANG Ke.Personalized top-N sequential recommendation via convolutional sequence embedding[C]//Proceedings of the Eleventh ACM International Conference on Web Search and Data Mining.[S1..]:[s.n.],2018:565-573.

[24] He X., Liao L., Zhang H., Nie L., Hu X., Chua T.-S.: Neural collaborative filtering. In Proceedings of WWW'17. 173-182 (2017).

[25] YANGLibin, ZHENGYu, CAIXiaoyan, etal. A LSTM based model for personalized context-aware citation recommendation[J].IEEE Access,2018,6:59618-59627.

[26] Huang P.-S., He X., Gao J., Deng L., Acero A., Heck L.: Learning deep structured semantic models for web search using click through data. In CIKM. 2333-2338 (2013).

[27] Liu P, Peng Z. China's smart city pilots: a progress report. Computer, 2014 (10): 72-81. 